\title{
ON $M_{0}$-SETS FOR SERIES WITH RESPECT TO CHARACTERS OF COMPACT ZERO-DIMENSIONAL GROUPS
}

\author{
VALENTIN SKVORTSOV
}

\begin{abstract}
Generalizing our previous result on Walsh system, we consider the system of characters of zero-dimensional compact abelian group. For this system, we give a construction of a perfect $M_{0}$-set whose Hausdorff $h$-measure equals zero, where $h$ is any non-decreasing right-continuous function taking the value zero at the origin.
\end{abstract}

\section{Introduction}

A set $E$ is said to be $U$-set for a system of functions (especially, for orthogonal system) if the convergence of a series with respect to this system to zero outside the set $E$ implies that all coefficients of the series are zero.

Otherwise, the set is called $M$-set, i.e., a set $E$ is said to be $M$-set for a system of functions if there exists a non-trivial series with respect to this system which converges to zero outside the set $E$. This series is called null-series if $\mu E=0$.

A set is called $M_{0}$-set, or a set of restricted multiplicity if it is an $M$-set and the corresponding non-trivial series is a Fourier-Stieltjes series of a probability measure concentrated on $E$.

D. E. Menshov constructed an $M_{0}$-set of measure zero for trigonometric series. Later, rather thin $M$-sets were constructed for other systems.

A good survey of the theory of sets of uniqueness and sets of multiplicity for the trigonometric system is given in [4].

Generalizing our previous result on Walsh system [7], we consider the system of characters of zero-dimensional compact abelian group and give, for this system, a construction of a perfect $M_{0}$-set whose Hausdorff $h$-measure equals zero,

(C) 2015 Mathematical Institute, Slovak Academy of Sciences.

2010 Mathematics Subject Classification: 42C10, 43A25, 43A75.

Keywords: compact zero-dimensional abelian group, characters of a group, $M$-set, $M_{0}$-set, Hausdorff $h$-measure.

Supported by RFBR (grant no. 14-01-00417) and by grant NSh-3682.2014.1. 


\section{VALENTIN SKVORTSOV}

where $h$ is any non-decreasing function which is right-continuous and taking the value zero at the origin. We apply our method from [7] which was also used in [2], where $M$-set of Haar measure zero for the system of characters of above mentioned group was constructed.

In Section 2, we recall some definitions and facts on the structure of a zero-dimensional compact abelian group and on the properties of the group of characters of this group. To justify the application of the method of [7] in the case of the group we are considering here, we need to extend to our case the theory of formal product of series, which was developed by Schneider in [6] in the case of Walsh system as an analogue of the Rajchman theory for the trigonometric case. We give this extension in Section 3. By this, we also justify results of [2], where such a justification was not presented. A construction of a perfect $M_{0}$-set is presented in Section 4 .

\section{Preliminaries}

Consider a zero-dimensional compact abelian group $G$. It is known (see [1]) that a topology in such a group can be given by a chain of subgroups

$$
G=G_{0} \supset G_{1} \supset \cdots \supset G_{n} \supset \cdots
$$

with $\{0\}=\bigcap_{n=0}^{+\infty} G_{n}$. The subgroups $G_{n}$ are clopen sets with respect to this topology. As $G$ is compact, the quotient groups $G / G_{n}$ are finite for each $n$. Let $p_{n}$ be the order of the quotient group $G_{n} / G_{n+1}$. Then, the order of $G_{0} / G_{1}$ is $p_{0}$, and the order of $G_{0} / G_{n}, n=1,2, \ldots$, is

$$
m_{n}=p_{0} \cdot p_{1} \cdots p_{n-1}
$$

with $p_{i} \geq 2$ for all $i=0,1,2, \ldots$ (we put $m_{0}=1$ ).

Typical examples of zero-dimensional compact abelian group are:

- Dyadic Cantor group $G$, i.e., the group of sequences of integers of the form $\tilde{x}=\left\{x_{j}\right\}_{j=0}^{\infty}$, where $0 \leq x_{j} \leq 1$ with group operation defined as component-wise addition modulo 2 for each component. Topology is defined by subgroups

$$
G_{n}=\left\{\tilde{x} \in G: x_{j}=0 \text { for } j=0, \ldots, n-1\right\} .
$$

- More general Vilenkin group $G$ is defined by a sequence of prime numbers $\left\{p_{j}\right\}, p_{j} \geq 2$. It is the group of sequences of integers of the form $\tilde{x}=\left\{x_{j}\right\}_{j=0}^{\infty}$, where $0 \leq x_{j} \leq p_{j}-1$ with group operation defined as component-wise addition modulo $p_{j}$ for $j$ th component. Topology is defined by the same type of subgroups as in the previous example. 


\section{ON $M_{0}$-SETS FOR SERIES WITH RESPECT TO CHARACTERS...}

- The additive group of $p$-adic integers (with $p$ being a fixed prime number) is the set of formal sums

$$
\sum_{j=0}^{\infty} x_{j} p^{j}
$$

where $0 \leq x_{i} \leq p-1$ and the addition is done with carries, i.e., to add two sequences $\sum_{j=0}^{\infty} x_{j} p^{j}$ and $\sum_{j=0}^{\infty} y_{j} p^{j}$, we add coordinate-wise, and if any of the sums is $p$ or more, we take a carry of 1 to the sum in the next coordinate. Topology is given by subgroups

$$
G_{n}=\left\{\sum_{j=0}^{\infty} x_{j} p^{j}: x_{j}=0 \text { for } j=1, \ldots, n-1\right\} .
$$

We denote any coset of the subgroup $G_{n}$ in $G / G_{n}$ by $K^{n}$.

It will be convenient to use the terminology of derivation basis theory (see, for example, [8]) and to refer to any $\operatorname{coset} K^{n}, n=0,1,2, \ldots$, as a $\mathcal{B}$-interval of rank $n$.

Let $\Gamma$ denote the dual group of $G$, i.e., the group of characters of the group $G$. It is known that under the assumption imposed on $G$, the group $\Gamma$ is a discrete abelian group with respect to the point-wise multiplication of characters (see [1]). Moreover, we can represent it as a union of increasing sequence of subgroups of finite order

$$
\Gamma_{0} \subset \Gamma_{1} \subset \Gamma_{2} \subset \cdots \subset \Gamma_{n} \subset \cdots
$$

Then, $\Gamma=\bigcup_{i=0}^{+\infty} \Gamma_{i}$ and $\Gamma_{0}$ consists of one character $\gamma_{0}$ with $\gamma_{0}(g)=1$ for all $g \in G$. For each $n \in \mathbb{N}$, the group $\Gamma_{n}$ is the annihilator of $G_{n}$, i.e.,

$$
\Gamma_{n}=G_{n}^{\perp}=\left\{\gamma \in \Gamma: \gamma(g)=1 \text { for all } g \in G_{n}\right\} .
$$

Note that in the case of the dyadic Cantor group, $\Gamma$ is the Walsh system and for the Vilenkin group it is the Price-Vilenkin system (see [3]).

The following lemma can be easily proved.

Lemma 2.1. If $\gamma \in \Gamma_{n}$, then $\gamma$ is constant on each coset $K^{n}$ of $G_{n}$.

Let $\mu$ be the Haar measure on the group $G$ normalized so that $\mu(G)=1$ which, in our case, can be constructed by the usual Lebesgue method starting with measure on the semi-ring composed by the family of all cosets $K^{n}$. The characters $\gamma$ constitute a countable orthonormal system on $G$ with respect to measure $\mu$. Note that $\mu\left(K^{n}\right)=1 / m_{n}$.

We can introduce a numeration of the characters in the following way.

The quotient groups $\Gamma_{n+1} / \Gamma_{n}=G_{n+1}^{\perp} / G_{n}^{\perp}$ and $G_{n} / G_{n+1}$ are isomorphic (see [1]) and so, they are of the same order $p_{n}$ for each $n \in \mathbb{N}$. This implies that the group $\Gamma_{n}$ has $m_{n}=p_{0} \cdot p_{1} \cdots p_{n-1}$ elements. 


\section{VALENTIN SKVORTSOV}

Having put $\gamma_{0}=1$, we pick up a character $\gamma \in \Gamma_{n+1} \backslash \Gamma_{n}$ for each $n=0,1,2, \ldots$ and denote it by $\gamma_{m_{n}}$. For

$$
n=\sum_{k=0}^{k_{n}} t_{k} m_{k}, \quad 0 \leq t_{k} \leq p_{k}-1
$$

define

$$
\gamma_{n}:=\prod_{k=0}^{k_{n}}\left(\gamma_{m_{k}}\right)^{t_{k}}
$$

This is the so-called Vilenkin-Paley numeration.

The functions $\gamma$ belonging to $\Gamma_{k} \backslash \Gamma_{k-1}$ are called the characters of rank $k$. In fact, $\gamma_{n}$ is of rank $k$ if $m_{k-1} \leq n \leq m_{k}-1$. According to Lemma 2.1, all the characters of rank $k$ are constant on $\mathcal{B}$-intervals of rank $k$. If $P$ is a polynomial with respect to $\Gamma$, then its rank is defined as the maximal rank of its terms.

Now, we can consider a series with respect to the system $\Gamma$ in the above numeration: $\sum_{i=0}^{\infty} a_{i} \gamma_{i}$ (coefficients $a_{n}$ are complex numbers).

An important subsequence of partial sums of this series is

$$
\sum_{i: \gamma_{i} \in \Gamma_{n}} a_{i} \gamma_{i}=S_{m_{n}}=\sum_{i=0}^{m_{n}-1} a_{i} \gamma_{i}
$$

\section{Formal product and its application}

Let $k(l, n)$ be an index defined by the equality $\gamma_{l} \gamma_{n}^{-1}=\gamma_{k(l, n)}$. In this notation, the product (formal) of a series $\sum_{n=0}^{\infty} a_{n} \gamma_{n}$ and a polynomial $\lambda=\sum_{n=0}^{p} b_{n} \gamma_{n}$ will mean the series $\sum_{l=0}^{\infty} c_{l} \gamma_{l}$, where $c_{l}=: \sum_{n=0}^{p} b_{n} a_{k(l, n)}$. This definition is justified by the formal equality

$$
\left(\sum_{n=0}^{p} b_{n} \gamma_{n}\right)\left(\sum_{k=0}^{\infty} a_{k} \gamma_{k}\right)=\sum_{l=0}^{\infty} \sum_{n=0}^{p} b_{n} a_{k(l, n)} \gamma_{n} \gamma_{k(l, n)}=\sum_{l=0}^{\infty} \sum_{n=0}^{p} b_{n} a_{k(l, n)} \gamma_{l} .
$$

Lemma 3.1. Let $n<m_{s}$, where $m_{s}$ is defined by (2.1), be fixed and let $k(l, n)$ be defined as above. Then, under the assumption that $q m_{s} \leq l<(q+1) m_{s}$ for some $q \in \mathbb{N}$, we have

$$
q m_{s} \leq k(l, n)<(q+1) m_{s}
$$

with the same $q$. Moreover, if $l \neq l^{\prime}$, then $k(l, n) \neq k\left(l^{\prime}, n\right)$.

P r o of. The last property is clear. Let

$$
l=\sum_{r=0}^{r_{l}} t_{r} m_{r}=\sum_{r=0}^{s-1} t_{r} m_{r}+\sum_{r=s}^{r_{l}} t_{r} m_{r}=l^{\prime}+l^{\prime \prime}, \quad \text { where } \quad 0 \leq t_{r}<p_{r} .
$$




\section{ON $M_{0}$-SETS FOR SERIES WITH RESPECT TO CHARACTERS...}

Note that $l^{\prime}<m_{s}$ and $l^{\prime \prime}=q m_{s}$ here. Then, according to (2.2), $\gamma_{l}=\gamma_{l^{\prime}} \gamma_{q m_{s}}$ with $\gamma_{l^{\prime}} \in G_{s}^{\perp}$.

The condition $n<m_{s}$ implies $\gamma_{n} \in G_{s}^{\perp}$ and so also $\gamma_{n^{\prime}}=: \gamma_{n}^{-1} \in G_{s}^{\perp}$, i.e., $n^{\prime}<m_{s}$. From this we get $\gamma_{k(l, n)}=\gamma_{l} \gamma_{n^{\prime}}=\gamma_{l^{\prime}} \gamma_{n^{\prime}} \gamma_{q m_{s}}$. With $\gamma_{l^{\prime}} \gamma_{n^{\prime}} \in G_{s}^{\perp}$ we have $\gamma_{l^{\prime}} \gamma_{n^{\prime}}=: \gamma_{k^{\prime}}$, where $0 \leq k^{\prime}<m_{s}$. Therefore by (2.2), $k(l, n)=q m_{s}+k^{\prime}$ and this implies (3.1).

TheOREM 3.1. Let a series

$$
\sum_{l=0}^{\infty} c_{l} \gamma_{l}
$$

be obtained as a formal product of the series

$$
\sum_{n=0}^{\infty} a_{n} \gamma_{n}, \quad \lim _{n \rightarrow \infty} a_{n}=0,
$$

and a polynomial $\lambda=\sum_{n=0}^{p} b_{n} \gamma_{n}$. Then the series (3.2) and

$$
\lambda \sum_{n=0}^{\infty} a_{n} \gamma_{n}
$$

are uniformly equi-convergent on $G$, i.e., the difference

$$
\lambda \sum_{n=0}^{N} a_{n} \gamma_{n}-\sum_{n=0}^{N} c_{n} \gamma_{n}
$$

converges to 0 uniformly on $G$ when $N \rightarrow \infty$.

Proof. Fix $s$ for which $p<m_{s}$ and note that for all $n \leq p$ and any $q \in \mathbb{N}$ we have

$$
\sum_{l=0}^{q m_{s}-1} a_{l} \gamma_{l}=\sum_{l=0}^{q m_{s}-1} a_{k(l, n)} \gamma_{k(l, n)} .
$$

Indeed, due to Lemma 3.1 inequality $l<q m_{s}$ implies $k(l, n)<q m_{s}$ and for different $l$ values of $k(l, n)$ are also different. So the right hand sum in the above equality is obtained from the left hand sum by a permutation of its terms. Therefore

$$
\begin{aligned}
\sum_{l=0}^{q m_{s}-1} c_{l} \gamma_{l}=\sum_{l=0}^{q m_{s}-1}\left(\sum_{n=0}^{p} b_{n} a_{k(l, n)}\right) \gamma_{l} & =\sum_{n=0}^{p} b_{n} \gamma_{n} \sum_{l=0}^{q m_{s}-1} a_{k(l, n)} \gamma_{k(l, n)} \\
& =\sum_{n=0}^{p} b_{n} \gamma_{n} \sum_{l=0}^{q m_{s}-1} a_{l} \gamma_{l}=\lambda \sum_{l=0}^{q m_{s}-1} a_{l} \gamma_{l}
\end{aligned}
$$




\section{VALENTIN SKVORTSOV}

For arbitrary $N$ we find $q$ so that $q m_{s} \leq N<(q+1) m_{s}$. Then

$$
\begin{aligned}
\left|\sum_{l=0}^{N} c_{l} \gamma_{l}-\lambda \sum_{l=0}^{N} a_{l} \gamma_{l}\right| & =\left|\sum_{l=q m_{s}}^{N} c_{l} \gamma_{l}-\lambda \sum_{l=q m_{s}}^{N} a_{l} \gamma_{l}\right| \\
& \leq \max _{q m_{s} \leq l<(q+1) m_{s}}\left|a_{l}\right| m_{s}\left(\sum_{n=0}^{p}\left|b_{k}\right|+\max _{g \in G}|\lambda(g)|\right) .
\end{aligned}
$$

The right-hand side converges to zero when $N$ together with $q$ tends to infinity.

TheOREM 3.2. For the series (3.3) and for any $\mathcal{B}$-interval $K^{n}$ there exists a series $\sum_{l=0}^{\infty} c_{l} \gamma_{l}$ with $\lim _{l \rightarrow \infty} c_{l}=0$ which is uniformly equi-convergent with (3.3) on $K^{n}$ and is uniformly convergent to zero outside $K^{n}$.

P r o of. The function $\chi_{K^{n}}$, the indicator of $K^{n}$, can clearly be represented as a polynomial $P$ with respect to $\Gamma$. Then, the formal product of $P$ and of the series (3.3) gives the series $\sum_{l=0}^{\infty} c_{l} \gamma_{l}$ we are looking for. Indeed, applying Theorem 3.1 to this polynomial, we get that series (3.3) is uniformly equi-convergent with the series $\chi_{K^{n}} \sum_{l=0}^{\infty} a_{l} \gamma_{l}$. However, this series is identical with series (3.3) on $K^{n}$ and is vanishing outside $K^{n}$.

We need the following version of the uniqueness theorem which is a particular case of [8, Corollary 4.3].

Theorem 3.3. If for the series (3.3) at each point $g \in G$ some subsequence of partial sums of the form $S_{m_{n_{k}}}(g)$ converges to zero, then all the coefficients of the series are equal zero.

With the above theorem we get

Theorem 3.4. If for the series (3.3) some subsequence of partial sums of the form $S_{m_{n_{k}}}(g)$ converges to zero for all $g$ on some open set $O$, then this series converges to zero on $O$.

Proof. As the system of all $\mathcal{B}$-intervals forms a basis of topology in $G$, it is sufficient to prove the statement for any $K^{n}$. We can apply Theorem 3.2 to the series (3.3) and to the chosen $K^{n}$. The subsequence of partial sums $S_{m_{n_{k}}}^{\prime}$ of the series

$$
\sum_{l=0}^{\infty} c_{l} \gamma_{l}
$$

given by Theorem 3.2 converges to zero everywhere on $G$. Now, by Theorem 3.3 , we have $c_{l}=0$ for all $l$. So, the series (3.6) having all coefficients zero is convergent to zero uniformly on $G$. Once again, applying Theorem 3.2 , we get that the series (3.3) is also convergent to zero on $K^{n}$. 


\section{ON $M_{0}$-SETS FOR SERIES WITH RESPECT TO CHARACTERS...}

\section{Construction of $M_{0}$-set}

Definition 4.1. Let $h$ be a nondecreasing right-continuous at zero function with $h(0)=0$ and let $\mu$ be the Haar measure on $G$. The Hausdorff $h$-measure $\mathcal{H}^{h}$ is defined by

$$
\mathcal{H}^{h}(A)=\liminf _{\delta \rightarrow 0} \sum\left\{h\left(\mu\left(E_{i}\right)\right): E_{i} \quad \mathcal{B} \text {-intervals, } \bigcup E_{i} \supset A, \mu\left(E_{i}\right) \leq \delta\right\} .
$$

TheOREM 4.1. For any $h$ as above, there exists a perfect $M_{0}$-set $E$ for the system $\Gamma$ whose Hausdorff h-measure equals zero.

The first step in the construction is the

Lemma 4.1. For any $A>0$ and any $\varepsilon>0$, there exists $N \in \mathbb{N}$ such that for any $K^{n}, n \geq N$, any $\delta>0$, any $B, 0<B \leq A$, and any $k>n, k \in \mathbb{N}$, there exists a polynomial

$$
P(g)=\sum_{j=m_{k}}^{m_{l}-1} a_{j} \gamma_{j}(g)
$$

with the following properties:

(1) $\left|a_{j}\right|<\varepsilon, m_{k} \leq j \leq m_{l}-1$;

(2) $P(g)=0$ if $g \in G \backslash K^{n}$;

(3) $P(g) \geq-B$ if $g \in K^{n}$;

(4) $\frac{m_{k}}{m_{n}} h\left(\frac{1}{m_{l}}\right)<\delta$,

where $\frac{m_{k}}{m_{n}}$ is the number of those $K^{l}$ in $K^{n}$ for which $P(g) \neq-B$ with $g \in K^{l}$.

Pr o of. Take $N$ such that $A / m_{N}<\varepsilon$ and having fixed arbitrary $n \geq N$ and $k>n$ choose $l$ so that

$$
\frac{m_{k}}{m_{n}} h\left(\frac{1}{m_{l}}\right)<\delta .
$$

Let $K^{n}$ be any $\mathcal{B}$-interval of rank $n$. It is a union of $m_{k} / m_{n} \mathcal{B}$-intervals of rank $k$. Let them be $K_{j}^{k}, j=1,2, \ldots, m_{k} / m_{n}$. Now, we choose and fix in each $K_{j}^{k}$ a $\mathcal{B}$-interval of rank $l$. Let it be $K_{j}^{l}$. Consider a function

$$
P(g):=\left\{\begin{array}{cl}
0 & \text { if } g \in G \backslash K^{n}, \\
-B & \text { if } g \in K_{j}^{k} \backslash K_{j}^{l}, \\
\left(m_{l} / m_{k}-1\right) B & \text { if } g \in K_{j}^{l} .
\end{array}\right.
$$

It is easy to show that this function can be represented as a polynomial of the form (4.1). Properties (2) and (3) follow directly from the definition of the function $P$, and property (4) follows from (4.2) and the choice of $K_{j}^{l}$. Property (1) was in fact checked in 2 . 


\section{VALENTIN SKVORTSOV}

Now, we sketch the proof of Theorem 4.1 once again following the scheme given in [2] and [7.

P r o of. Having taken a sequence

$$
\left\{\varepsilon_{k}\right\}, \varepsilon_{k}>0, \lim _{k \rightarrow \infty} \varepsilon_{k}=0,
$$

we construct by induction a sequence of polynomials $\left\{Q_{k}\right\}$ with respect to $\Gamma$ having the following properties:

(1) $Q_{1}(g)=1$ for all $g \in G$;

(2) maximal rank $s_{k}$ of the characters $\gamma$ constituting the polynomial $Q_{k}$ is less than the minimal rank of those constituting $Q_{k+1}$;

(3) coefficients $a_{i}$ of the polynomial $Q_{k}$ satisfy the inequality $\left|a_{i}\right| \leq \varepsilon_{k}$;

(4) $\sum_{i=1}^{k} Q_{i}(g) \geq 0$ for any $g \in G$;

(5) $\sum_{i=1}^{k} Q_{i}(g)=0$ for $g \in G \backslash E_{k}$, where $E_{k}=\bigcup_{j=1}^{j_{k}} I_{j}, j_{k} \geq 2^{k-1}$ with $I_{j}$ being $\mathcal{B}$-intervals of various ranks greater than $k$ (starting with $k=2$ ) so that

$$
\sum_{j=1}^{j_{k}} h\left(\mu\left(I_{j}\right)\right)<\varepsilon_{k} \text { and } E_{k+1} \subset E_{k} .
$$

Construction of polynomial $Q_{k+1}$ can be realized by a repeated application of Lemma 4.1 to $\mathcal{B}$-intervals constituting $E_{k}$ following the lines of the proof of [7, Theorem 1].

Property (2) implies that the sum $\sum_{k=1}^{\infty} Q_{k}$ defines a series

$$
\sum_{i=0}^{\infty} a_{i} \gamma_{i}
$$

with partial sums

$$
S_{m_{s_{k}}}=\sum_{i=1}^{k} Q_{i}
$$

By property (3), $\lim _{i \rightarrow \infty} a_{i}=0$. Property (5) implies that the Hausdorff $h$-measure of the set $E=\bigcap_{k=1}^{\infty} E_{k}$ is zero. It is clear from the construction that $E$ is a perfect set in $G$. By the same property, the partial sums (4.4) tend to zero on $G \backslash E$ and this set is open. So, we can apply Theorem 3.4 to this set to obtain that the series (4.3) converges to zero outside $E$.

To prove that this null-series is a Fourier-Stieltjes series, we define a $\mathcal{B}$-interval function by putting for each interval of rank $s_{k}, k=1,2, \ldots$,

$$
F\left(K^{s_{k}}\right)=\int_{K^{s_{k}}} S_{m_{s_{k}}}(g) d \mu .
$$




\section{ON $M_{0}$-SETS FOR SERIES WITH RESPECT TO CHARACTERS...}

It can be easily checked that $F$ defines an additive function on the family of all $\mathcal{B}$-intervals. It is usually referred to as a quasi-measure associated with the series (see [9]). By property (4), $F$ is non-negative, by property (1),

$$
F(G)=\int_{G} S_{m_{0}}(g) d \mu=1
$$

and by property $(5), F(I)=0$ for any $\mathcal{B}$-interval $I \subset G \backslash E$. So, $F$ generates a probability measure $\mu_{F}$ on $G$ supported by $E$, and for any $\mathcal{B}$-interval $I$ we have $\mu_{F}(I)=F(I)$. Let $i<m_{n}$ and let $\gamma_{i}\left(K_{j}^{n}\right), j=1,2, \ldots, m_{n}$, denote the constant values which $\gamma_{i}$ assumes on $K_{j}^{n}$. Then,

$$
\begin{aligned}
a_{i}=\int_{G} S_{m_{n}} \gamma_{i} d \mu & =\sum_{j=1}^{m_{n}} \gamma_{i}\left(K_{j}^{n}\right) F\left(K_{j}^{n}\right) \\
& =\sum_{j=1}^{m_{n}} \gamma_{i}\left(K_{j}^{n}\right) \mu_{F}\left(K_{j}^{n}\right)=\int_{G} \gamma_{i} d \mu_{F} .
\end{aligned}
$$

So, the series (3.4) is the Fourier-Stieltjes series of the measure $\mu_{F}$ and $E$ is an $M_{0}$-set.

In the conclusion, we note that $\mathrm{P}$ i at etski - $\mathrm{Sh}$ a pir o gave an example of a closed $M$-set for the trigonometric system which is not an $M_{0}$-set (see [4]). An analogous result for the Vilenkin system is obtained in [5]. It is an open problem whether such an example can be constructed in the general case of the system of characters we are considering here, and, in particular, for the system of characters of the group of $p$-adic integers.

\section{REFERENCES}

[1] AGAEV, G. N.-VILENKIN, N. YA.-DZHAFARLI, G. M.-RUBinshtein, A. I.: Multiplicative system of functions and harmonic analysis on zero-dimensional groups. "Ehlm", Baku, 1981. (In Russian)

[2] BOKAEV, N. A.-NURKHANOV M. A.: Example of a null-series with respect to periodic multiplicative systems, Math. Notes 54 (1993), 1187-1191.

[3] GOLUBOV, B.-EFIMOV, A.-SKVORTSOV, V.: Walsh series and transforms: Theory and Applications, in: Math. Appl., Vol. 64, Kluwer Acad. Publ., Dordrecht, 1991.

[4] KECHRIS, A. S.-LOUVEAU, A.: Descriptive set theory and the structure of sets of uniqueness, London Math. Soc. Lecture Note Ser., Vol. 128, Cambridge Univ. Press, 1989.

[5] LIPPMAN, G. E.-WADE, W. R.: Pseudofunctions and uniqueness on the group of integers of a p-series field, Acta Math. Acad. Sci. Hungar. 35 (1980), 1-12.

[6] SCHNEIDER, A. A.: On the uniqueness of expansions in Walsh functions, Mat. Sbornik 24 (1949), 279-300. 


\section{VALENTIN SKVORTSOV}

[7] SKVORTSOV, V. A.: The h-measure of M-sets for the Walsh system, Mat. Zametki 21 (1977), 335-340; Engl. transl. in Math. Notes 21 (1977), 186-189.

[8] SKVORTSOV, V. A.-TULONE, F.: Kurzweil-Henstock type integral in Fourier analysis on compact zero-dimensional group, Tatra Mt. Math. Publ. 44 (2009), 41-51.

[9] WADE, W. R.-YONEDA, K.: Uniqueness and quasi-measure on the group of integers of p-series field, Proc. Amer. Math. Soc. 84 (1982), 202-206.

Received November 28, 2014

Institute of Mathematics

Casimirus the Great University

pl. Weyssenhoffa 11

PL-85-079 Bydgoszcz

POLAND

Department of Mathematics

Moscow State University

Moscow 119991

RUSSIA

E-mail:vaskvor2000@yahoo.com 